\title{
COMMENT ON: "DO SUNSPOTS MATTER WHEN SPOT MARKET EQUILIBRIA ARE UNIQUE?"
}

\author{
By Richard C. BARNETT AND ERIC O’N. FISHER ${ }^{1}$
}

MAS-COLELL (1992) CONJECTURED that there could be no sunspot equilibria if the fundamentals of the underlying economy admitted a unique equilibrium. Hens (2000) constructed an example to show that this conjecture was false. A weak form of Mas-Colell's conjecture is indeed false, but there is an error in Hens's proof. The first part of this comment shows the mistake in Hens's argument, and the second part proves the existence of the relevant sunspot equilibria. We conclude with a discussion of why an example like Hens's is necessary if one wishes to prove that a stricter form of Mas-Colell's conjecture is indeed false.

We follow Hens's notation almost exactly. There are two sunspot-contingent assets paying returns denominated in units of the numeraire. These returns are

$$
V=\left[\begin{array}{ll}
0 & 1 \\
b & 0 \\
c & 0
\end{array}\right]
$$

where $v_{s j}$ is the return on asset $j$ in state $s$. There are two households indexed by $i \in\{1,2\}$, and in each state of nature there are two goods. The fundamentals of the economy are endowments $\omega^{1}=(1,0)^{\prime}$ and $\omega^{2}=(0,1)^{\prime}$ and preferences described by the composite function $h^{i} \circ u^{i}: R_{+}^{2} \rightarrow R$. The function $u^{i}(\cdot)$ has the rule $u^{i}\left(x^{i}\right)=\left(x_{1}^{i}\right)^{\alpha^{i}}\left(x_{2}^{i}\right)^{1-\alpha^{i}}$ and $h^{i}(\cdot)$ is assumed to be differentiable, strictly monotone, and concave.

The probability that state $s$ occurs is $\pi(s)$, and the von Neumann-Morgenstern expected utility indicators are $U^{i}\left(x^{i}\right)=\sum_{s=1}^{3} \pi(s) h^{i} \circ u^{i}\left(x^{i}(s)\right)$. Let $p(s)=\left(p_{1}(s), p_{2}(s)\right)^{\prime}$. Then, taking spot market prices $p=\left(p(1)^{\prime}, p(2)^{\prime}, p(3)^{\prime}\right)^{\prime}$ and asset prices $q=\left(q_{1}, q_{2}\right)^{\prime}$ as given, each agent chooses a consumption plan $x^{i} \in R_{+}^{6}$ and asset demands $y^{i} \in R^{2}$ to maximize $U^{i}\left(x^{i}\right)$ subject to $q \cdot y^{i} \leq 0$ and, for $s \in S=\{1,2,3\}, p(s) \cdot x^{i}(s) \leq p(s) \cdot \omega^{i}+V(s) y^{i}$, where $V(s)$ is row $s$ of the asset return matrix $V$. An equilibrium is a list of spot market prices $p$, asset prices $q$, and corresponding allocations $\left(x^{i}\right)_{i=1}^{2}$ and asset demands $\left(y^{i}\right)_{i=1}^{2}$ such that $x^{i}$ and $y^{i}$ solve the household's problem, $\sum_{i} x^{i}(s) \leq \sum_{i} \omega^{i}$ for $s \in S$, and $\sum_{i} y^{i} \leq 0$. The sunspot equilibrium is nontrivial if for some agent $x^{i}(s) \neq x^{i}\left(s^{\prime}\right)$ for some $s, s^{\prime} \in S$.

Let $v^{i}\left(p(s), w^{i}(s)\right)$ be the indirect utility of agent $i$ facing spot market prices $p(s)$ and having ex post income $w^{i}(s)=p(s) \cdot \omega^{i}+V(s) y^{i}$. Since each ex post spot market has a unique equilibrium, we can write $h^{i}(s)=h^{i} \circ v^{i}\left(p(s), w^{i}(s)\right)$ without ambiguity. For ease of exposition, we follow Hens and put $h^{1}(\cdot)=h(\cdot), h^{2}(\cdot)=g(\cdot), \alpha^{1}=\alpha$, and $\alpha^{2}=\beta$. Using Hens's four normalizations $q_{2}=1$ and $p_{2}(s)=1$, we see that the asset demands imply

$$
\left[\begin{array}{ccc}
q_{1} h^{\prime}(1) / p_{1}(1)^{\alpha} & -b h^{\prime}(2) / p_{1}(2)^{\alpha} & -c h^{\prime}(3) / p_{1}(3)^{\alpha} \\
q_{1} g^{\prime}(1) / p_{1}(1)^{\beta} & -b g^{\prime}(2) / p_{1}(2)^{\beta} & -c g^{\prime}(3) / p_{1}(3)^{\beta} \\
1 & 1 & 1
\end{array}\right]\left[\begin{array}{c}
\pi(1) \\
\pi(2) \\
\pi(3)
\end{array}\right]=\left[\begin{array}{l}
0 \\
0 \\
1
\end{array}\right] .
$$

${ }^{1}$ The authors thank Thorsten Hens for helpful comments. 
Since expected utility is defined up to an affine transformation, we can always set $q_{1} h^{\prime}(1) / p_{1}(1)^{\alpha}=q_{1} g^{\prime}(1) / p_{1}(1)^{\beta}=1$ and then write

$$
\left[\begin{array}{ccc}
1 & -b h^{\prime}(2) / p_{1}(2)^{\alpha} & -c h^{\prime}(3) / p_{1}(3)^{\alpha} \\
1 & -b g^{\prime}(2) / p_{1}(2)^{\beta} & -c g^{\prime}(3) / p_{1}(3)^{\beta} \\
1 & 1 & 1
\end{array}\right]\left[\begin{array}{c}
\pi(1) \\
\pi(2) \\
\pi(3)
\end{array}\right]=\left[\begin{array}{l}
0 \\
0 \\
1
\end{array}\right] .
$$

Write (1) as $A \pi=(0,0,1)^{\prime}$; we are looking for a solution satisfying $\pi \gg 0$. Note that

$$
\begin{aligned}
A^{-1}= & (1 / \operatorname{det} A) \\
& \times\left[\begin{array}{ccc}
\cdots & \cdots & b c\left[\left(h^{\prime}(2) / p_{1}(2)^{\alpha}\right)\left(g^{\prime}(3) / p_{1}(3)^{\beta}\right)-\left(g^{\prime}(2) / p_{1}(2)^{\beta}\right)\left(h^{\prime}(3) / p_{1}(3)^{\alpha}\right)\right] \\
\cdots & \cdots & -c\left[h^{\prime}(3) / p_{1}(3)^{\alpha}-g^{\prime}(3) / p_{1}(3)^{\beta}\right] \\
\cdots & \cdots & b\left[h^{\prime}(2) / p_{1}(2)^{\alpha}-g^{\prime}(2) / p_{1}(2)^{\beta}\right]
\end{array}\right] .
\end{aligned}
$$

Since $\pi=A^{-1}(0,0,1)^{\prime}, \pi \gg 0$ only if all the elements in the last column of $A^{-1}$ have the same sign.

Hens sets $y_{1}^{1}=-1, \alpha=1 / 4, \beta=1 / 2,-1<b<0$, and $1>q_{1}>c>0$. These assumptions imply that $p_{1}(1)=\left(2-q_{1}\right) / 3, p_{1}(2)=(b+2) / 3$, and $p_{1}(3)=(c+2) / 3$. Since $b, q_{1}, c \in(-1,1), p_{1}(1) \in(1 / 3,2 / 3), p_{1}(2) \in(1 / 3,2 / 3)$, and $p_{1}(3) \in(2 / 3,1)$. Thus $p_{1}(2)<p_{1}(3)$ and $p_{1}(1)<p_{1}(3)$. The normalizations imply that state-dependent indirect utilities are $v^{1}\left(p(s), w^{1}(s)\right)=\alpha^{\alpha}(1-\alpha)^{1-\alpha} w^{1}(s) / p_{1}(s)^{\alpha}$ and $v^{2}\left(p(s), w^{2}(s)\right)=\beta^{\beta}(1-$ $\beta)^{1-\beta} w^{2}(s) / p_{1}(s)^{\beta}$. Note that $w^{1}(1) / p_{1}(1)^{\alpha}=(2 / 3)\left(1+q_{1}\right) / p_{1}(1)^{\alpha}, w^{1}(2) / p_{1}(2)^{\alpha}=$ $(2 / 3)(1-b) / p_{1}(2)^{\alpha}$, and $w^{1}(3) / p_{1}(3)^{\alpha}=(2 / 3)(1-c) / p_{1}(3)^{\alpha}$. Also, $w^{2}(1) / p_{1}(1)^{\beta}=(1-$ $\left.q_{1}\right) / p_{1}(1)^{\beta}, w^{2}(2) / p_{1}(2)^{\beta}=(1+b) / p_{1}(2)^{\beta}$, and $w^{2}(3) / p_{1}(3)^{\beta}=(1+c) / p_{1}(3)^{\beta}$. Computation shows that $w^{1}(1) / p_{1}(1)^{\alpha}>w^{1}(3) / p_{1}(3)^{\alpha}$ and $w^{1}(2) / p_{1}(2)^{\alpha}>w^{1}(3) / p_{1}(3)^{\alpha}$ and also that $w^{2}(1) / p_{1}(1)^{\beta}<w^{2}(3) / p_{1}(3)^{\beta}$ and $w^{2}(2) / p_{1}(2)^{\beta}<w^{2}(3) / p_{1}(3)^{\beta}$. Since $g(\cdot)$ and $h(\cdot)$ are both strictly increasing and concave, $0<h^{\prime}(1) \leq h^{\prime}(3), 0<h^{\prime}(2) \leq h^{\prime}(3)$, $g^{\prime}(1) \geq g^{\prime}(3)>0$, and $g^{\prime}(2) \geq g^{\prime}(3)>0$.

If all three elements in that column are negative, then in particular the first one is. Since $b c<0,\left(h^{\prime}(2) / p_{1}(2)^{\alpha}\right)\left(g^{\prime}(3) / p_{1}(3)^{\beta}\right)>\left(g^{\prime}(2) / p_{1}(2)^{\beta}\right)\left(h^{\prime}(3) / p_{1}(3)^{\alpha}\right)$ and thus $h^{\prime}(2) g^{\prime}(3) / h^{\prime}(3) g^{\prime}(2)>p_{1}(3)^{\beta-\alpha} / p_{1}(2)^{\beta-\alpha}>1$, contradicting that $h^{\prime}(2) g^{\prime}(3) / h^{\prime}(3) g^{\prime}(2) \leq$ 1. If all three elements in that column are positive, then in particular the second one is. Since $c>0, h^{\prime}(3) / p_{1}(3)^{\alpha}<g^{\prime}(3) / p_{1}(3)^{\beta}$ and thus $h^{\prime}(3) / g^{\prime}(3)<1 / p_{1}(3)^{\beta-\alpha}$. Since we have imposed that $q_{1} h^{\prime}(1) / p_{1}(1)^{\alpha}=q_{1} g^{\prime}(1) / p_{1}(1)^{\beta}=1, g^{\prime}(1) / h^{\prime}(1)=p_{1}(1)^{\beta-\alpha}$ and thus $h^{\prime}(3) g^{\prime}(1) / g^{\prime}(3) h^{\prime}(1)<p_{1}(1)^{\beta-\alpha} / p_{1}(3)^{\beta-\alpha}<1$, where the last inequality follows from $p_{1}(1)<p_{1}(3)$. But this contradicts the fact that $h^{\prime}(3) g^{\prime}(1) / g^{\prime}(3) h^{\prime}(1) \geq 1$. Thus Hens's example is incorrect.

Where exactly did Hens go wrong? Like us, he uses the first-order necessary conditions characterizing the two agents' asset demands to form a linear system in the probabilities of the sunspot states. Solving the system, he establishes three restrictions on some auxiliary functions that ensure that the probability of each state is strictly positive. His proof is constructive, and he attempts to show the existence of strictly concave functions that satisfy a system of inequalities. He actually relies on piecewise linear approximations to more general concave functions. Using these approximations, he describes properties of the auxiliary functions ((i)-(v) of Hens (2000)) that he says suffice for the requisite inequalities to hold. Unfortunately, his five conditions do not imply his original inequalities. In particular, condition (v) is not strong enough. 
We will now construct an equilibrium where sunspots matter. The matrix for sunspotcontingent asset payoffs is

$$
V=\left[\begin{array}{ll}
1 & 1 \\
1 & 0 \\
0 & 1
\end{array}\right] .
$$

For simplicity, we impose that $\pi(1)=\pi(2)=\pi(3)=1 / 3$ and use the same normalizations as before. Let endowments again be $\omega^{1}=(1,0)^{\prime}$ and $\omega^{2}=(0,1)^{\prime}$. Preferences are now represented by $u^{1}\left(x^{1}\right)=\left(x_{1}^{1}\right)^{1 / 2}\left(x_{2}^{1}\right)^{1 / 2}$ and $u^{2}\left(x^{2}\right)=\left(x_{1}^{2}\right)^{1 / 2}\left(x_{2}^{2}\right)^{1 / 2}$, with $h^{1}(u)=u$ and $h^{2}(u)=u$. Now the spot prices are $p_{1}(s)=1$ for all $s \in S .^{2}$ Since the indirect utility functions for the two agents are identical, the asset demand conditions show that $q_{1}=1$.

Now consider either agent's problem. He takes prices as given and chooses $y^{i}$ to maximize expected utility subject to $q^{1} y_{1}^{i}+y_{2}^{i} \leq 0$. Since $q_{1}=1$, it is easy to see that any $0 \leq y_{1}^{i} \leq 1$ maximizes expected utility and satisfies the nonnegativity constraints. If $y_{1}^{i} \neq 0$, then sunspots matter because the equilibrium allocations are random, even though the supporting prices are not. Since each agent's ex ante expected utility is unchanged, these equilibria are quite trivial. It is still an open question whether this admittedly simple example can be extended to the case where the agents are strictly risk averse and have preferences that are not identical and homothetic.

We conclude by arguing that the following four conditions are necessary for MasColell's conjecture to be false in economies where spot prices differ across sunspot realizations and where agents are risk averse. First, there must be at least two agents so that there can be some ex ante asset trade. Second, there must be at least two assets for exactly the same reason. Third, there must be at least three states of nature so that markets are actually incomplete, and Cass and Shell's (1983) classic result about full insurance is not germane. Fourth, there must be at least two goods in each spot market, since otherwise no risk-averse agent would hold a strictly positive amount of any asset even if it were priced at fair odds.

Dept. of Economics, Southern Methodist University, Box 0496, Room 301J, Umphrey Lee Bldg., 3300 Dyer St., Dallas, TX 75275-0496, U.S.A.; rbarnett@mail.smu.edu and

Dept. of Economics, The Ohio State University, 410 Arps Hall, 1945 N. High St., Columbus, OH 43210, U.S.A.; fisher.244@osu.edu; http://economics.sbs.ohio-state.edulefisher

Manuscript received July, 2000; final revision received December, 2000.

\section{REFERENCES}

CASs, DAvid, AND K. SHell (1983): “Do Sunspots Matter?” Journal of Political Economy, 91, 193-227.

${ }^{2}$ The unique no-sunspot equilibrium has $p_{1}(s)=1, q_{1}=1$, and $x^{1}(s)=(1 / 2,1 / 2)^{\prime}, x^{2}(s)=$ $(1 / 2,1 / 2)^{\prime}$, and $y^{1}=y^{2}=(0,0)^{\prime}$. Because preferences are identical and homothetic, there is only one price vector that supports equilibrium for any $\omega^{1}+\omega^{2}=(1,1)^{\prime}$. Thus the equilibria are distinguished only by virtue of their allocations, not supporting prices. 
Hens, Thorsten (2000): “Do Sunspots Matter when Spot Markets are Unique?” Econometrica, 68, 435-441.

MAs-Colell, Andreu (1992): "Three Observations on Sunspots and Asset Redundancy," in Economic Analyses of Markets and Games: Essays in Honor of Frank Hahn, ed. by P. Dasgupta, D. Gale, O. Hart, and E. Maskin. Cambridge, MA: MIT Press. 